\title{
FT-NIR: a tool for rapid intracellular lipid quantification in oleaginous yeasts
}

\author{
Mikołaj Chmielarz ${ }^{1}$, Sabine Sampels ${ }^{1}$, Johanna Blomqvist ${ }^{2}$, Jule Brandenburg ${ }^{1}$, Frida Wende ${ }^{1}$, Mats Sandgren ${ }^{1}$
} and Volkmar Passoth ${ }^{1 *}$ (D)

\begin{abstract}
Background: Lipid extraction for quantification of fat content in oleaginous yeasts often requires strong acids and harmful organic solvents; it is laborious and time-consuming. Therefore, in most cases just endpoint measurements of lipid accumulation are performed and kinetics of intracellular lipid accumulation is difficult to follow. To address this, we created a prediction model using Fourier-transform near-infrared (FT-NIR) spectroscopy. This method allows to measure lipid content in yeast.

Methods: The FT-NIR calibration sets were constructed from spectra of freeze-dried cells of the oleaginous yeasts Rhodotorula toruloides CBS 14, Lipomyces starkeyi CBS 1807 and Yarrowia lipolytica CBS 6114. The yeast cells were obtained from different cultivation conditions. Freeze-dried cell pellets were scanned using FT-NIR in the Multi Purpose Analyser (MPA) from Bruker. The obtained spectra were assigned corresponding to total fat content, obtained from lipid extraction using a modified Folch method. Quantification models using partial least squares (PLS) regression were built, and the calibration sets were validated on independently cultivated samples. The $R$. toruloides model was additionally tested on Rhodotorula babjevae DBVPG 8058 and Rhodotorula glutinis CBS 2387.
\end{abstract}

Results: The $R^{2}$ of the FT-NIR model for $R$. toruloides was $98 \%$, and the root mean square error of cross-validation (RMSECV) was 1.53. The model was validated using a separate set of $R$. toruloides samples with a root mean square error of prediction (RMSEP) of 3.21. The $R^{2}$ of the Lipomyces model was $96 \%$, with RMSECV 2.4 and RMSEP 3.8. The $R^{2}$ of the mixed model, including all tested yeast strains, was 90.5\%, with RMSECV 2.76 and RMSEP 3.22, respectively. The models were verified by predicting the total fat content in newly cultivated and freeze-dried samples. Additionally, the kinetics of lipid accumulation of a culture were followed and compared with standard lipid extraction methods.

Conclusions: Using FT-NIR spectroscopy, we have developed a faster, less laborious and non-destructive quantification of yeast intracellular lipid content compared to methods using lipid extraction.

Keywords: FT-NIR, Lipid quantification, Rhodotorula toruloides, Lipomyces starkeyi, Yarrowia lipolytica

\section{Background}

Lipids from renewable sources are of particular interest due to their potential use as a sustainable feedstock for biodiesel and chemicals production, and as ingredients in food or animal feed [1-6]. Oleaginous yeasts can accumulate lipids to more than $20 \%$ of their dry weight. The basidiomycetous red yeast Rhodotorula toruloides has

\footnotetext{
*Correspondence: Volkmar.Passoth@slu.se

${ }^{1}$ Department of Molecular Sciences, Swedish University of Agricultural

Sciences, Box 7015, 75007 Uppsala, Sweden

Full list of author information is available at the end of the article
}

the ability to utilise and accumulate lipids using glycerol, glucose and xylose as sole carbon sources. Thus, $R$. toruloides has the potential to convert side products such as lignocellulose from forest and agricultural production systems, or crude glycerol from biodiesel transesterification, into lipids of higher value. There are also ascomycetous oleaginous yeasts of interest, e.g. Lipomyces starkeyi that is known for its high lipid content and potential for fuel production [2], and the nonpathogenic dimorphic aerobic yeast Yarrowia lipolytica, for its highly developed tool box for genetic modification, for instance, to yield 
high lipid content or the production of long-chain $\Omega-3$ fatty acids [3].

One of the biggest challenges in optimising yeastbased lipid production systems is the ability to monitor lipid accumulation in yeast cells during their cultivation. Lipid extraction from microbes usually relies on organic solvents, often in the presence of strong acids and at elevated temperatures to break the cell walls [5-8]. Organic solvents are hazardous for the environment and to human health. Moreover, because extraction is performed in a biphasic system, errors can be introduced to lipid quantification due to pipetting errors. These errors can, however, be kept small by extracting lipids from large sample volumes. This can, however, affect experimental fermentations, since the total fermentation volume can be significantly changed during the time course of the cultivation by the removal of large sample volumes. Apart from this, lipid extraction is also labour-intensive and time-consuming. For these reasons, lipid production is in most cases monitored by endpoint measurement methods [7, 8], and the kinetics of lipid accumulation has rarely been investigated.

Infrared spectroscopy (IR) measures the absorption of light by fundamental molecular vibrations. It can be used to identify biomolecules such as proteins, lipids, DNA and other organic compounds [9]. Near-infrared (NIR) spectroscopy measures absorption of light vibrational overtones and their combinations. The NIR region of the electromagnetic wavelength spectrum ranges from $780 \mathrm{~nm}$ (wave number $12,800 \mathrm{~cm}^{-1}$ ) to about $2500 \mathrm{~nm}$ $\left(4000 \mathrm{~cm}^{-1}\right)$. At wavelengths above $2500 \mathrm{~nm}$, the midIR region starts. Both IR and NIR spectroscopy have a number of useful applications, in industry and within research, for the identification and quantification of different organic compounds, product quality control and authentication [10-12]. These spectroscopic methods have also been applied for yeast species identification and strain differentiation [13, 14]. NIR spectroscopy among other methods is used to analyse sugar and lipid content in the bioethanol industry [15]. The absorption bands in NIR spectroscopy are broad and often overlapping, resulting in complex spectra that are difficult to interpret [16]. In classical NIR spectroscopy, the sample is scanned by a monochromatic light which is sequentially changed to different wavelengths over the whole NIR wavelength bandwidth. The principle of Fourier-transform (FT)-NIR is slightly different: a sample is exposed to polychromatic light, which is then divided at the beam splitter resulting in a so-called interferogram showing the intensity of the light as a function of time. The interferogram is then transformed to the frequency domain by Fourier transformation. The advantage of FT-NIR compared to classical NIR spectroscopy is that it is faster, as it allows simultaneous measurement over the whole wavelength range, is more sensitive as there is a lower background noise, and has a higher precision [16]. Both IR and NIR spectroscopy techniques have been used before, for example, to analyse lipids in the human body in a noninvasive way [17], lipid content and type in plants [18], to determine olive oil quality [19], or to analyse lipids in milk [20], yeasts [21, 22] and moulds [23]. FT-NIR could hence also be a possible method to analyse yeast cell total fat content in a non-destructive way without the need for prior lipid extraction and the use of organic solvents.

The spectra produced by NIR spectroscopy contain a lot of information. It is sensitive to compound concentrations, physical structure, water content, etc. Since the NIR absorption bands are so broad and overlapping, the whole spectra or selected regions are selected for analysis. Multivariate analysis such as partial least squares (PLS) regression is often used to produce predictive models. A set of NIR calibration spectra are assigned to reference values obtained from available analytical methods [24]. The aim of this study was to establish a rapid non-destructive FT-NIR method for lipid quantification in oleaginous yeasts and additionally to minimise the usage of organic solvents used in conventional lipid concentration determination methods.

\section{Results}

Pellets of yeast cells grown on different carbon sources at different cultivation times (see "Materials and methods") were collected and analysed further. From the obtained spectra, a lipid prediction model was generated for $R$. toruloides.

For the FT-NIR calibration set, 60 unique samples of $R$. toruloides were analysed in triplicates, from which 179 spectra were used, followed by lipid extraction to provide a reference value. As an external validation set, 18 samples were collected and analysed in the same way. The FT-NIR wavenumber regions of interests for lipid concentration measurements were the following: $4167-4545 \mathrm{~cm}^{-1}, 5600-6150 \mathrm{~cm}^{-1}, 6900-7300 \mathrm{~cm}^{-1}$ and $8000-9000 \mathrm{~cm}^{-1}$. At these regions, $\mathrm{C}-\mathrm{H}$ bonds are absorbing infrared light [25].

The determined fat content in the analysed $R$. toruloides samples varied between 5.9 and 59\% of the cell dry weight (Figs. 1, 2).

An FT-NIR quantification model for $R$. toruloides total fat content was generated using the OPUS software included in the FT-NIR software package provided by the instrument manufacturer, and this FT-NIR quantification model was compared with the fat content data that were obtained from lipid extraction (Fig. 1). The calibration set produced for the $R$. toruloides cells with different lipid concentrations resulted in an FT-NIR quantification 
model with a cross-validation $R^{2}$ value of $98 \%$ and a RMSECV value of 1.53 at rank 9. Vector normalisation was used for spectra pre-processing. The FT-NIR regions used to produce the model were: $8562.9-8038.4 \mathrm{~cm}^{-1}$ and $4485.9-4069.3 \mathrm{~cm}^{-1}$. The linear regression equation was: $y=0.9726 x+0.9786$. Model accuracy was confirmed by comparing the lipid content of the samples predicted by the model with results obtained from the lipid extractions (Figs. 3 and 4). Additionally, separate test samples were used for external validation of the model showing that the produced model had an RMSEP value of 3.21. Samples were obtained from cultures grown in hemicellulose hydrolysate, mixed hydrolysate, crude glycerol or glucose with an addition of yeast nitrogen base (YNB). Figure 1 shows that the FT-NIR lipid predictions were not influenced by the medium composition. The yeast lipid accumulation data were compared with the prediction model data (see Fig. 4). In this comparison, the average difference between real data and predicted lipid content was $5.2 \%$. To see whether the functioning $R$. toruloides model works on different yeast species, we tested the model on Lipomyces starkeyi samples, which resulted in large prediction errors (Fig. 5).

In a similar fashion as for the model for $R$. toruloides, spectra and analytical data for L. starkeyi were used to

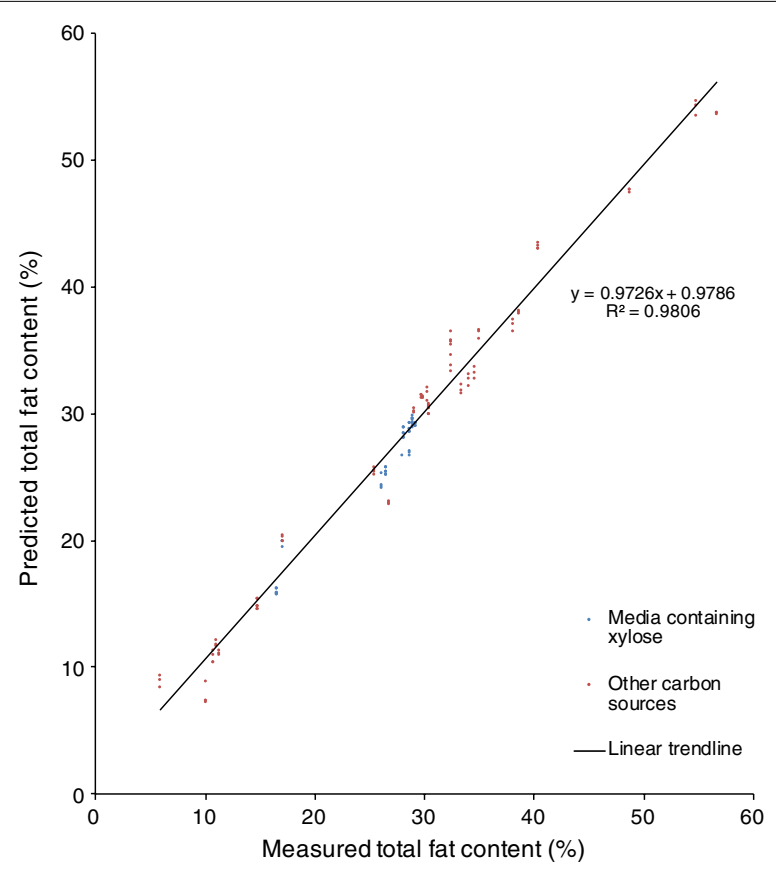

Fig. 1 Cross-validation of the total fat content in R. toruloides CBS 14 . From cultures grown in hemicellulose hydrolysate, mixed hydrolysate, crude glycerol or glucose with YNB. Blue dots represent cultures grown in media containing xylose as the main carbon source; red dots represent cultures grown in media containing other carbon sources (glycerol and glucose) create a Lipomyces lipid FT-NIR quantification model. The Lipomyces model consisted of 99 calibration spectra, with a $R^{2}$ of $96 \%$ (Fig. 6) and a RMSECV of 2.4 at rank 2. Vector normalisation was used for spectra pre-processing, and FT-NIR regions used were: $8694.1-8061.5 \mathrm{~cm}^{-1}$, $7151.2-6649.8 \mathrm{~cm}^{-1}$ and $4466.6-3849.5 \mathrm{~cm}^{-1}$. The linear regression equation was: $y=0.94 x+1.54$. To test the model, 98 spectra from L. starkeyi strains (CBS 1807, CBS 7544), and Yarrowia lipolytica CBS 6114 were used with a resulting validation RMSEP of 3.8 (Fig. 7).

Spectra and analytical data of $R$. toruloides, $L$. starkeyi and Y. lipolytica were used to create a combined lipid FTNIR quantification model. The combined model consisted of 238 calibration spectra, with a $R^{2}$ of $90.5 \%$ (Fig. 2) and a RMSECV of 2.76. The second derivative was used for spectra pre-processing, and FT-NIR regions used were: $8775.1-8034.5 \mathrm{~cm}^{-1}$ and $6001.8-5554.3 \mathrm{~cm}^{-1}$. The linear regression equation was: $y=0.9158 x+1.8607$. To test the combined model, 55 spectra, including additionally R. glutinis CBS 2387 and R. babjevae DBVPG 8058, were used to test elasticity of the FT-NIR model with a resulting validation RMSEP of 3.22.

We compared predictions of lipid concentrations based on the combined model and the Lipomyces model with values determined by lipid extraction on four independent L. starkeyi CBS 1807 samples. The Lipomyces model

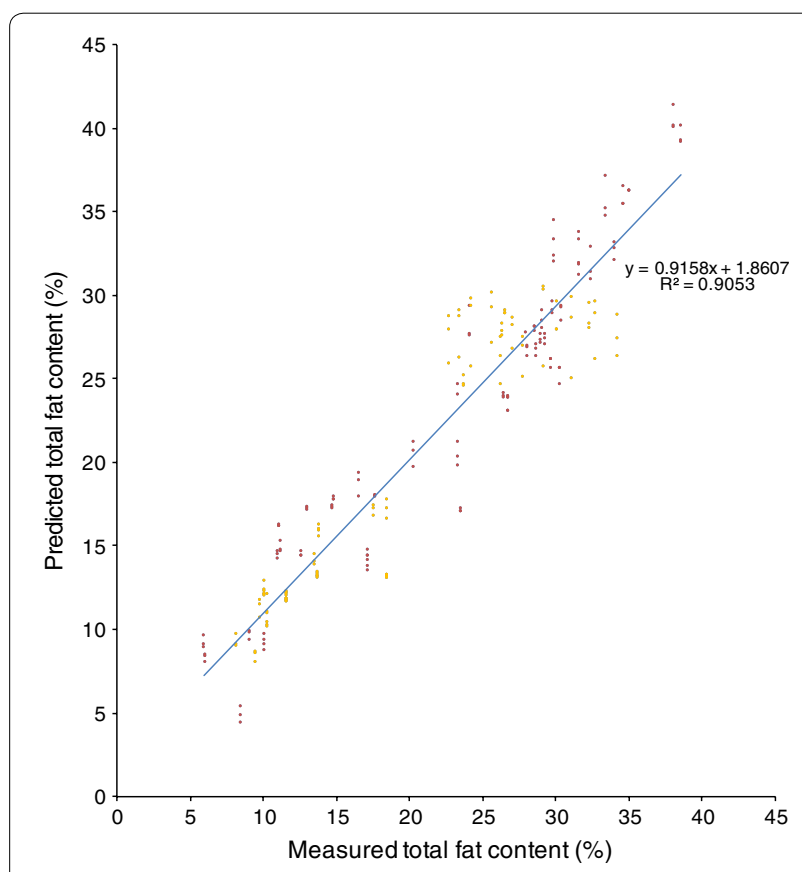

Fig. 2 Cross-validation of the total fat content in the model of several combined yeast species (R. toruloides, L. starkeyi, Y. lipolytica), cultivations using hemicellulose hydrolysate, mixed hydrolysate, crude glycerol or glucose with YNB as cultivation media. Red dots represent red yeast; yellow dots represent L. starkeyi and Y. lipolytica samples 


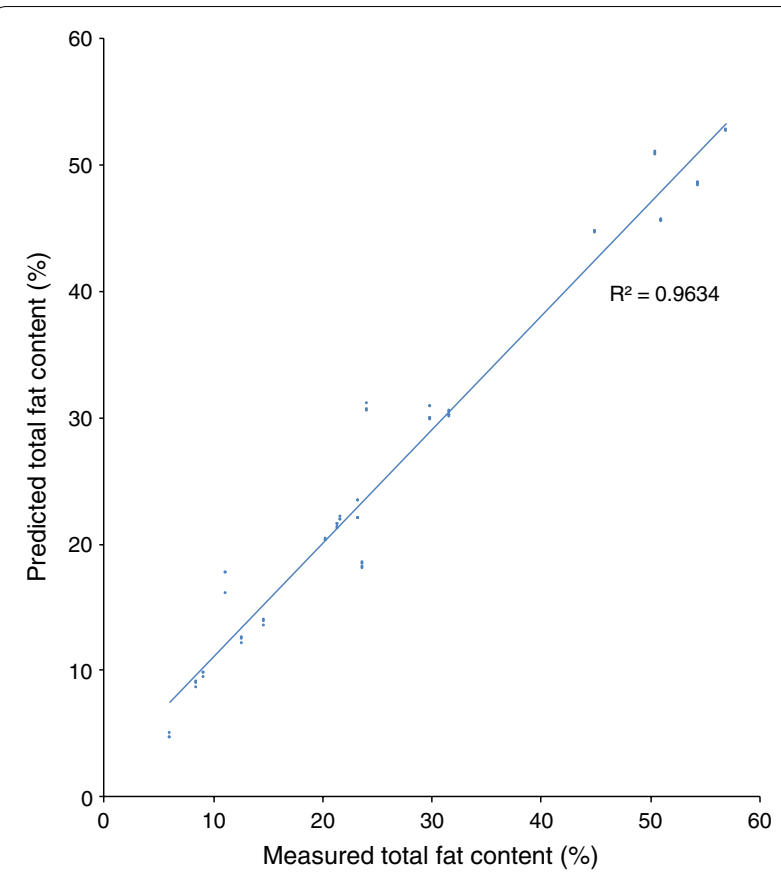

Fig. 3 Test set validation of the total fat content in R. toruloides CBS 14 model. Cells were grown using YNB media with glucose, xylose and glycerol. A total 161 spectra were used for the calibration set of the model. Independent samples in form of 42 spectra were used as test set validation values were more similar to the lipid extraction values than the combined model predictions (Fig. 8).

\section{Discussion}

In the present study, we prove that it is possible to rapidly measure lipid content in red (Rhodotorula sp.) and other (Lipomyces starkeyi, Yarrowia lipolytica) yeast cells using FT-NIR spectroscopy with a high accuracy. It was only necessary to use organic solvent for lipid extractions during the establishment of the FT-NIR model, and afterwards they were only needed when clear outliers were present, or to further refine the FT-NIR model with new model data points. The verification of the produced FT-NIR lipid model on samples collected during cultivation of $R$. toruloides CBS 14 on different growth media, especially those taken during cultivation on lignocellulosic hydrolysate, further indicates that different cultivation methods do not substantially influence the FT-NIR-based lipid concentration predictions, as long as the analysed yeast cells are washed prior to the FT-NIR measurements. The FT-NIR model can also be used for analysing samples taken during cultivations using "difficult" cultivation media, to follow the lipid accumulation in the culture over time. In the previous studies, Laurens et al. established a lipid content model by using NIR

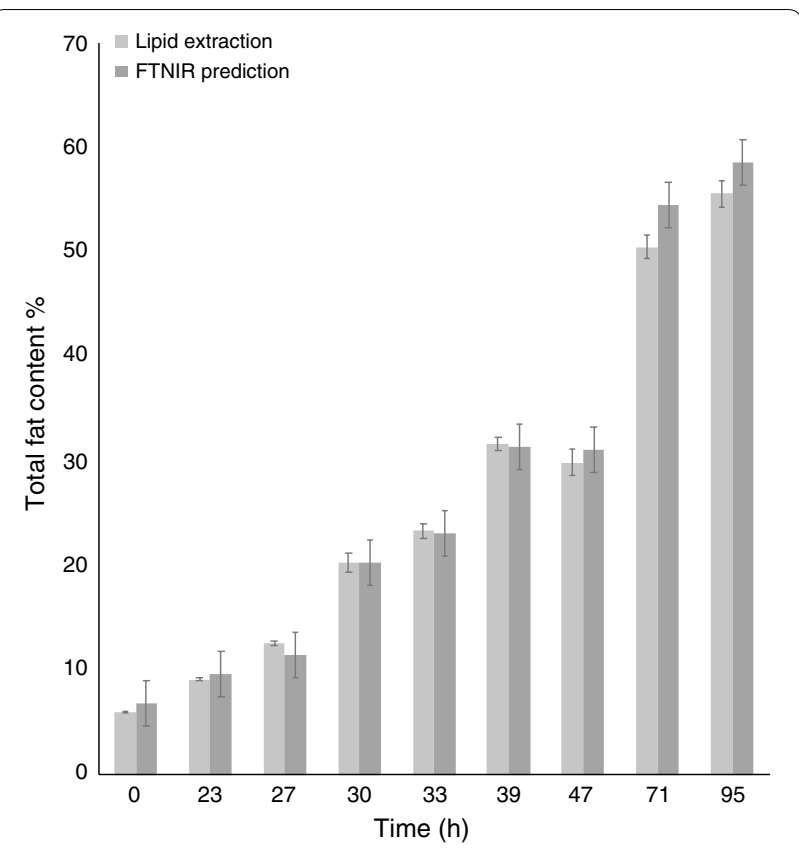

Fig. 4 Comparison of Rhodotorula accuracy (dark grey bar) with traditional lipid extraction (light grey). Values were obtained by analysing test culture samples using the Quant2 analysis package. The samples were obtained from the cultivation of $R$. toruloides cultivated in a lignocellulosic hydrolysate $(n=27)$. The error in prediction was 2.17 for the FT-NIR model. Average difference between the lipid extraction results and the FT-NIR predictions was $5.2 \%$

measurements [21]. However, they only tested standard cultivation media, and used nitrogen limitation to get higher variation in lipid accumulation. In our study, we used different types of media, including lignocellulose hydrolysate, which, due to its complexity, could create problems if resulting in a variation in the lipid composition of the cells. In food science, it has been shown, for example, that it was possible to detect differences in the lipid composition due to variation of the cultivation conditions (e.g. region or temperature) [26, 27]. In contrast to this, our results clearly show that the cultivation media did not cause a significant variation in the lipid detection in yeast samples.

Laurens et al. [21] used a similar approach as in our study, but applied a different apparatus and processing of spectra. In particular, they used fatty acid methyl esters (FAME) instead of total fat. Unfortunately, both methods cannot directly be compared, as raw measuring data have to be processed by the according transformations.

It was not possible to use our existing red yeast model for the prediction of lipid content in non-pigmented yeasts. Similarly, the Lipomyces model could not be used for determining lipids in red yeasts. However, it was 


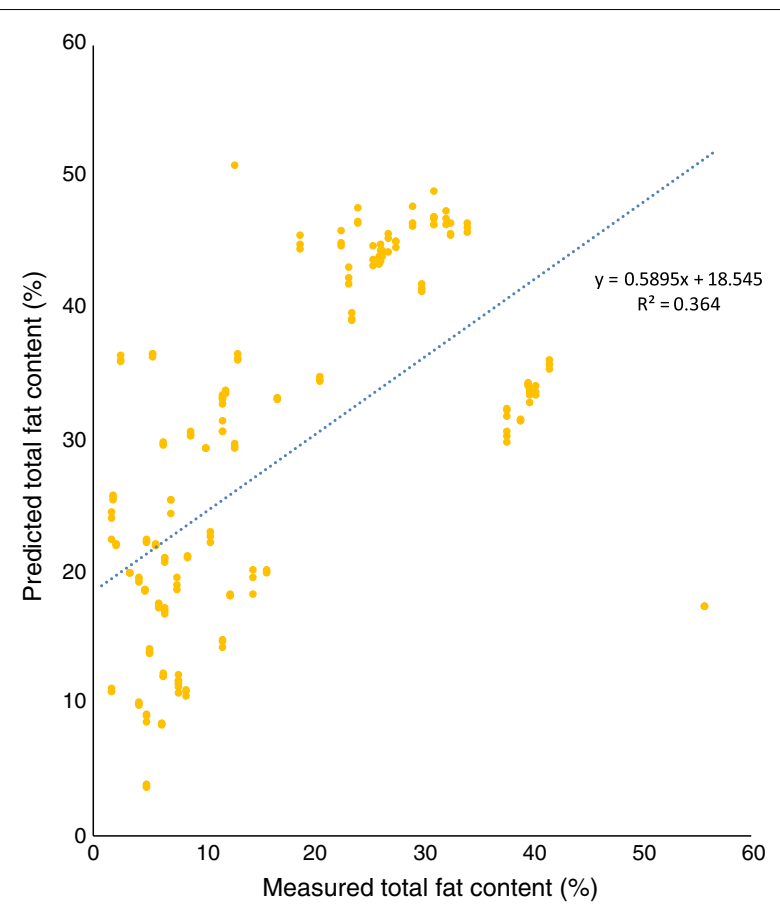

Fig. 5 Using prediction model of R. toruloides on spectra of L. starkeyi, cultured in YNB with the addition of glycerol or glucose

possible to use the Lipomyces model on Y. lipolytica. It needs to be tested on a per-case basis whether a strain belonging to another species can be analysed using a specific model. However, our results show that it is possible to combine species from different phyla of fungi when creating a model (Figs. 2, 8), in accordance with the results by Laurens et al. [21]. Compared to their model, our combined model was of acceptable quality. However, the model itself and the accuracy of it can be improved further by continuously adding additional samples [12, 25].

An advantage of using an FT-NIR-based lipid concentration determination method is that it is possible to share the model and data between different groups and users, thereby making it possible to expand the produced models by adding more samples from various sources. The data used to produce the prediction model are not restricted to one type of FT-NIR machine. This is already being done in, e.g., the bioethanol industry when measuring starch content in grains from different farms and harvest years, and at different production facilities. The models used by the industries are based on results from research activities on FT-NIR analysis of starch content in grains [28]. A future goal could be to establish an open access FT-NIR database used by academic groups carrying out research on lipid accumulating yeasts and industries focusing on yeast lipid production systems.

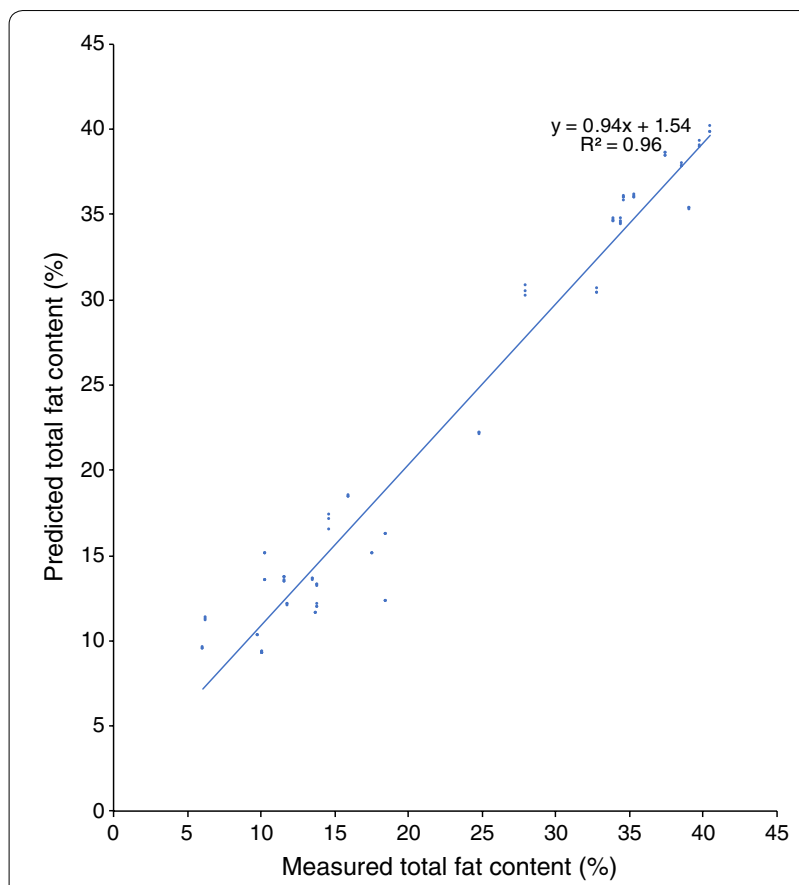

Fig. 6 Cross-validation of the total fat content in the Lipomyces model of L. starkeyi, CBS 1807, cultivations using glycerol or glucose with YNB as cultivation media

It has previously been pointed out and discussed that it can be difficult to create reliable and robust FT-NIR calibration models due to necessary knowledge of chemometrics [12]. In case of the OPUS software, which was used in our study, establishing and expanding models requires only basic knowledge and can be relatively rapidly performed. This simplifies the use of the model, and it is also possible to improve model accuracy by adding additional data points in the future. It should be noted that it is not required to use FT-NIR hardware for data processing (only for spectra collection), so the model itself can be analysed offline.

In the present study, the sample volumes used for classical lipid extraction and the measurement carried out by FT-NIR were approximately the same. However, it is possible to use smaller FT-NIR probes and thereby reduce the sample volume used [21,29]. This is another advantage, especially when following lipid accumulation during the whole fermentation process when cell density is low, e.g. at the beginning of the cultivation, or during smallscale testing or screening.

We have created FT-NIR models to determine lipid content that is easy to implement for end user and that can be easily shared thanks to the possibility of exporting the model and sample values to other types of FT-NIR equipments with compatible software. As mentioned earlier, other studies tried different approaches with FT-IR 


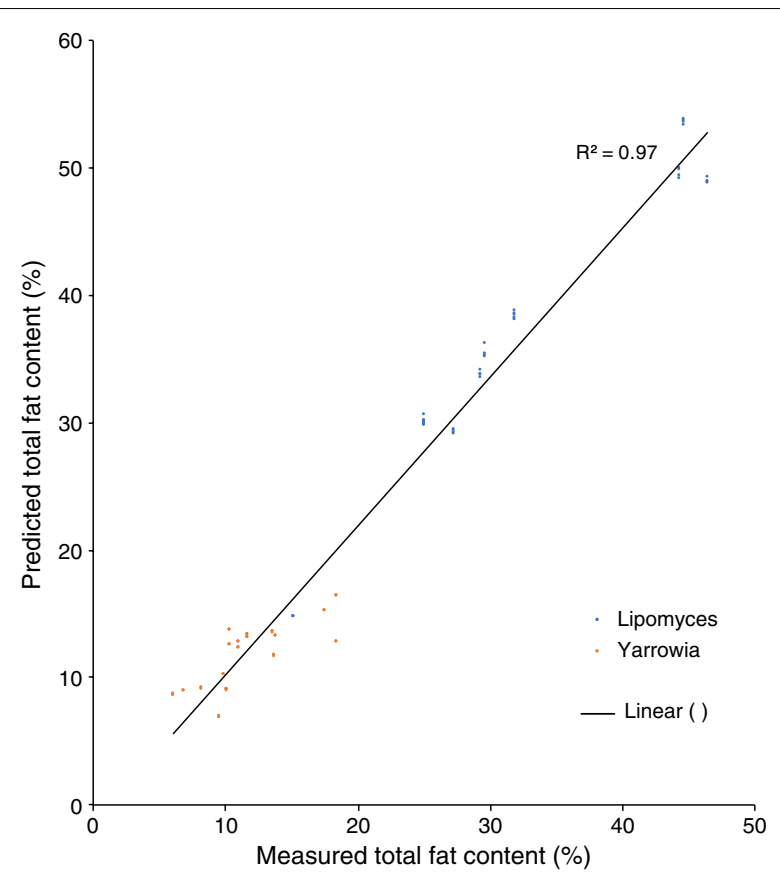

Fig. 7 Test set validation of the total fat content in the Lipomyces model. Cells were grown using YNB media with glucose, xylose and glycerol. In total, 98 spectra from L. starkeyi and Y. lipolytica were used for the test set validation

and NIR for lipid detection [21,22]. Both solutions are viable alternatives to our approach. With the FT-NIR method in combination with a dedicated software, a narrower spectra band than IR is used and most of the statistical operation is automated and performed without the user input. In all methods, freeze-dried sample is preferred, but wet yeast cells should be possible to be analysed, too, because there are examples of measuring fat in water solution like milk [20]. In the future, the technique could be also used to evaluate lipid classes and/or, fatty acid composition and level of oxidation in addition to total fat content similarly as described in $[12,21]$.

\section{Conclusions}

To the best of our knowledge, we present for the first time working FT-NIR models for quantification of total fat content in oleaginous yeasts as fast and non-destructive alternatives for lipid extraction. These new models drastically reduce the time needed to obtain total fat content values, from few hours to a matter of minutes per sample analysed. Although gravimetric lipid determination is still more precise than prediction of the lipid content by the model, FT-NIR analyses will enable high-throughput measurements of lipid formation and kinetics studies of intracellular lipid accumulation. The precision of the model prediction can be increased further by including a growing number of new calibration values into the model.

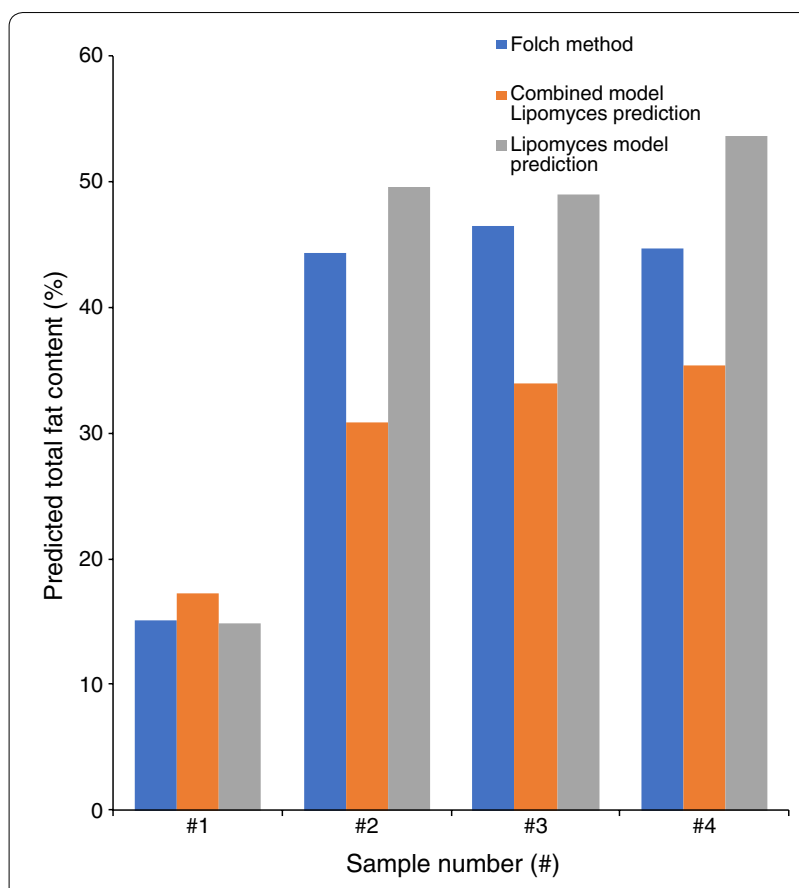

Fig. 8 Comparison between combined model and Lipomyces model prediction to values obtained with Folch extraction on four samples of Lipomyces starkeyi CBS 1807

\section{Materials and methods}

\section{Yeast strains and cultivation conditions}

The yeast strains used were $R$. toruloides CBS 14, L. starkeyi CBS 1807, L. starkeyi CBS 7544, Y. lipolytica CBS 6114, R. glutinis CBS 2387 (obtained from Centraalbureau voor Schimmelcultures, Utrecht, The Netherlands) and $R$. babjevae DBVPG 8058 (Industrial Yeast Collection, Perugia, Italy originally strain number J195, strain collection of the Department of Molecular Sciences, Swedish University of Agricultural Sciences, isolated from apple). They were stored at $-80{ }^{\circ} \mathrm{C}$ in $50 \%$ glycerol stock media. Before usage, the strains were transferred onto Yeast extract-Malt extract (YM) plates-10 g/L glucose ( $\geq 99 \%$, Fluka Analytical, France), 5 g/L peptone (from casein, Merck KGaA, Germany), $3 \mathrm{~g} / \mathrm{L}$ Yeast extract $^{\left(\text {Bacto }^{\text {TM }}\right.}$ Yeast Extract, BD, France), $3 \mathrm{~g} / \mathrm{L}$ malt extract (Merck KGaA, Germany), $16 \mathrm{~g} / \mathrm{L}$ agar (VWR Chemicals, Sweden). After 3 days of growth at $25^{\circ} \mathrm{C}$, the agar plates were stored at $4{ }^{\circ} \mathrm{C}$. Pre-cultures were grown in YPD medium, in shake flasks at $25^{\circ} \mathrm{C}$ for $72 \mathrm{~h}$.

All media components except for yeast nitrogen base (YNB) and lignocellulose hydrolysates were autoclaved at $121{ }^{\circ} \mathrm{C}$ for $20 \mathrm{~min}$. YNB and hydrolysate were sterilefiltered with a $0.2-\mu \mathrm{m}$ filter (VWR International, LLC, USA).

All experimental media were based on YNB medium with additional salts: $1.7 \mathrm{~g} / \mathrm{L}$ YNB (Difco ${ }^{\mathrm{TM}}$ yeast nitrogen 


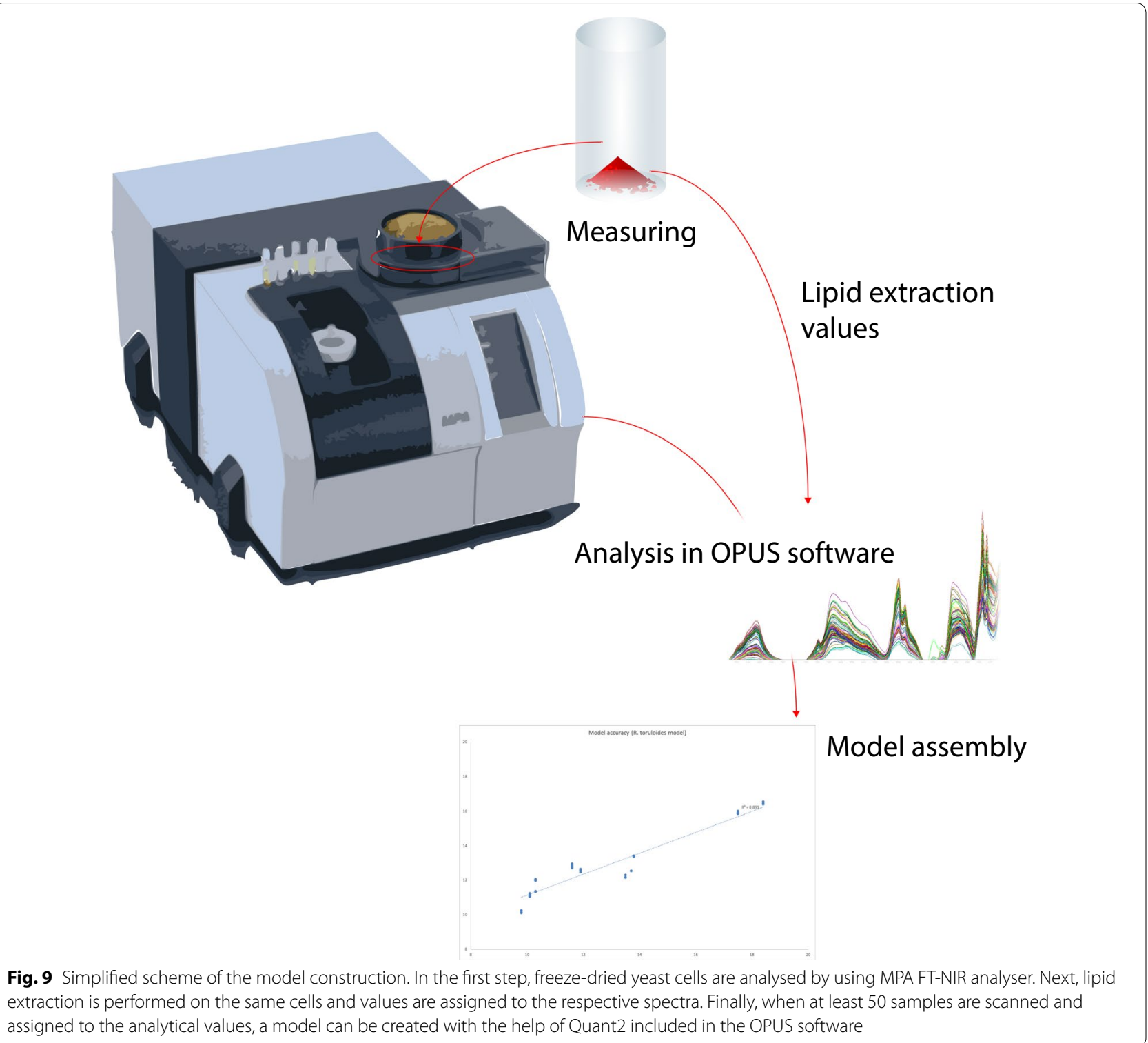

base w/o Amino acids and Ammonium Sulphate, BD, France), $0.75 \mathrm{~g} / \mathrm{L}$ Yeast extract (Bacto $^{\mathrm{TM}}$ Yeast Extract, BD, France), $2 \mathrm{~g} / \mathrm{L}\left(\mathrm{NH}_{4}\right)_{2} \mathrm{HPO}_{4}(\geq 98 \%$, Sigma-Aldrich, USA), $\mathrm{MgCL}_{2} 1 \mathrm{~g} / \mathrm{L}$ (Merck KGaA, Germany). The following carbon sources and additives were used: crude glycerol from biodiesel production $(850 \mathrm{~g} / \mathrm{L}$ glycerol $(85 \%$ purity), provided by Perstorp AB), glucose (10-30 g/L), xylose $(10-30 \mathrm{~g} / \mathrm{L})$, acetic acid $(0.5-3 \mathrm{~g} / \mathrm{L}, 95-97 \%$, Sigma-Aldrich, USA), hemicellulose hydrolysate (from wheat straw-up to $40 \% \mathrm{v} / \mathrm{v}$ of hydrolysate used in experiments), cellulose hydrolysate (from wheat strawup to $60 \% \mathrm{v} / \mathrm{v}$ of hydrolysate used in experiments). The hemicellulose/cellulose hydrolysate mixture carbon sources were determined by HPLC (Agilent 1100 Series,
Germany) $50.8 \mathrm{~g} / \mathrm{L}$ glucose, $29.3 \mathrm{~g} / \mathrm{L}$ xylose and $5.2 \mathrm{~g} / \mathrm{L}$ acetic acid. Wheat straw hydrolysate was obtained by steam explosion as described in Blomqvist et al. [2].

Yeast cultivations were performed in 2-L bioreactors (Minifors, Infors HT, Switzerland), $500 \mathrm{~mL}$ (Multifors, Infors HT, Switzerland) and in $100-\mathrm{mL}$ baffled shake flasks (Werner-Glas, Sweden). The cultivations in bioreactors were performed with the following parameters: $\mathrm{pH}=6\left(\right.$ acid $3 \mathrm{M} \mathrm{H}_{3} \mathrm{PO}_{4}$, base $\left.5 \mathrm{M} \mathrm{NaOH}\right), \mathrm{DO}=21 \%$ $\mathrm{O}_{2}$, and temperature $=25^{\circ} \mathrm{C}$. Cultivations in shake flasks conditions: initial $\mathrm{pH}=6$, cultivation time $72 \mathrm{~h}$, shaking at $130 \mathrm{rpm}, 25^{\circ} \mathrm{C}$ (Ecotron, Infors HT, Switzerland).

Each of the collected cultivation samples was washed with water two times and freeze-dried for $72 \mathrm{~h}$ in 
$-100{ }^{\circ} \mathrm{C}$ (CoolSafe Scanvac, LaboGene ApS, Denmark) to remove water and other possible compounds that can interfere with lipids in the spectra. After the collection of spectra, lipids from cells were extracted and quantified using a modified Folch method as described earlier [8].

A multi-purpose analyser (MPA) FT-NIR spectrometer from Bruker equipped with sample compartment and integrating sphere, combined with OPUS [OPUS ver. 7.5 build: 7.5.18 (20140810)], was used to obtain and analyse all FT-NIR spectra. All measurements were done in triplicates by using the integrated sphere compartment with the following parameters: Quartz beam splitter, RT_PbS detector, resolution of scan $16 \mathrm{~cm}^{-1}$, background measured internally. Glass vials containing freeze-dried cell pellets were scanned 32 times. Spectra were collected in the wavelength range from 4000 to $10,000 \mathrm{~cm}^{-1}$. A prediction model was generated with PLS regression using OPUS software Quant2 tool.

To ensure accuracy of the FT-NIR model, a smaller independent test set of samples, separate from calibration, were cultivated and analysed in the same manner. These samples were used to compare model prediction with actual extraction results.

The measuring procedure is illustrated in Fig. 9.

\begin{abstract}
Abbreviations
IR: infrared; NIR: near infrared; FAME: fatty acid methyl esters; FT-IR: Fouriertransform infrared; FT-NIR: Fourier-transform near infrared; RMSECV: root mean square error of cross-validation; RMSEP: root mean square error of prediction; PLS: partial least square; MPA: multi-purpose analyser; YNB: yeast nitrogen base; YM: yeast extract-malt extract; YPD: yeast peptone dextrose; HIP: hexane isopropanol.
\end{abstract}

\section{Acknowledgements}

The authors gratefully acknowledge Irina Ehnström for providing Lipomyces yeast samples.

\section{Authors' contributions}

MC was involved in designing the study, performing the major part of the laboratory work, model creation and writing the first draft of the manuscript. SS was involved in laboratory work, evaluation of the results and performed a major contribution to writing. JBI contributed to laboratory work, evaluation of the results and writing. JBr and FW contributed in evaluating the results and writing the manuscript. MS and VP provided a major contribution to design the study, to evaluate the results and to write the manuscript. All authors read and approved the final manuscript.

\section{Funding}

The research was supported by The Swedish Research Council for Environment, Agricultural Sciences and Spatial Planning (Formas), Grant Numbers 213-2013-80 and 2018-01877.

\section{Availability of data and materials}

The datasets used and/or analysed during the current study are available from the corresponding author on reasonable request.

Ethics approval and consent to participate

Not applicable.

\section{Consent for publication}

All authors agreed to publish this article.

\section{Competing interests}

The authors declare that they have no competing interests.

\section{Author details}

${ }^{1}$ Department of Molecular Sciences, Swedish University of Agricultural Sciences, Box 7015, 75007 Uppsala, Sweden. ${ }^{2}$ Faculty of Science and Technology, Norwegian University of Life Sciences: NMBU, P.O.Box 5003, 1432 Ås, Norway.

Received: 5 March 2019 Accepted: 21 June 2019

Published online: 29 June 2019

\section{References}

1. Petrovic U. Next-generation biofuels: a new challenge for yeast. Yeast. 2015;32(9):583-93.

2. Blomqvist J, Pickova J, Tilami SK, Sampels S, Mikkelsen N, Brandenburg J, et al. Oleaginous yeast as a component in fish feed. Sci Rep. 2018;8(1):15945.

3. Passoth V. Lipids of yeasts and filamentous fungi and their importance for biotechnology. In: Sibirny AA, editor. Biotechnology of yeasts and filamentous fungi. Cham: Springer International Publishing; 2017. p. 149-204.

4. Karlsson H, Ahlgren S, Sandgren M, Passoth V, Wallberg O, Hansson PA. A systems analysis of biodiesel production from wheat straw using oleaginous yeast: process design, mass and energy balances. Biotechnol Biofuels. 2016:9:229.

5. Karlsson H, Ahlgren S, Sandgren M, Passoth V, Wallberg O, Hansson PA Greenhouse gas performance of biochemical biodiesel production from straw: soil organic carbon changes and time-dependent climate impact. Biotechnol Biofuels. 2017;10:217.

6. Passoth $\vee$, Sandgren M. Biofuel production from straw hydrolysates: current achievements and perspectives. Appl Microbiol Biotechnol. 2019;103(13):5105-16.

7. Brandenburg J, Poppele I, Blomqvist J, Puke M, Pickova J, Sandgren M, et al. Bioethanol and lipid production from the enzymatic hydrolysate of wheat straw after furfural extraction. Appl Microbiol Biotechnol. 2018;102(14):6269-77.

8. Brandenburg J, Blomqvist J, Pickova J, Bonturi N, Sandgren M, Passoth V. Lipid production from hemicellulose with Lipomyces starkeyi in a $\mathrm{pH}$ regulated fed-batch cultivation. Yeast. 2016;33(8):451-62.

9. Tamm LK, Tatulian SA. Infrared spectroscopy of proteins and peptides in lipid bilayers. Q Rev Biophys. 1997;30(4):365-429.

10. Dalle Zotte A, Berzaghi P, Jansson L-M, Andrighetto I. The use of near-infrared reflectance spectroscopy (NIRS) in the prediction of chemical composition of freeze-dried egg yolk and discrimination between different n-3 PUFA feeding sources. Anim Feed Sci Technol. 2006;128(1-2):108-21.

11. Duan C, Zhang J-F, Hu Y, Zeng L, Su D, Bao G-M. A distinctive near-infrared fluorescence turn-on probe for rapid, sensitive and chromogenic detection of sulfite in food. Dyes Pigm. 2019;162:459-65.

12. Xu J-L, Riccioli C, Sun D-W. An overview on nondestructive spectroscopic techniques for lipid and lipid oxidation analysis in fish and fish products. Compr Rev Food Sci Food Saf. 2015;14(4):466-77.

13. Rellini P, Roscini L, Fatichenti F, Morini P, Cardinali G. Direct spectroscopic (FTIR) detection of intraspecific binary contaminations in yeast cultures. FEMS Yeast Res. 2009;9(3):460-7.

14. Santos C, Fraga ME, Kozakiewicz Z, Lima N. Fourier transform infrared as a powerful technique for the identification and characterization of filamentous fungi and yeasts. Res Microbiol. 2010;161(2):168-75.

15. Beekes M, Lasch P, Naumann D. Analytical applications of Fourier transform-infrared (FT-IR) spectroscopy in microbiology and prion research. Vet Microbiol. 2007;123(4):305-19.

16. Stuart BH. Infrared spectroscopy: fundamentals and applications. New York: Wiley; 2004.

17. Azizian H, Kramer JK, Heymsfield SB, Winsborough S. Fourier transform near infrared spectroscopy: a newly developed, non-invasive method to 
measure body fat: non-invasive body fat content measurement using FT-NIR. Lipids. 2008;43(1):97-103.

18. Camps C, Gerard M, Quennoz M, Brabant C, Oberson C, Simonnet X. Prediction of essential oil content of oregano by hand-held and Fourier transform NIR spectroscopy. J Sci Food Agric. 2014;94(7):1397-402.

19. Mossoba MM, Azizian H, Fardin-Kia AR, Karunathilaka SR, Kramer JKG. First application of newly developed FT-NIR spectroscopic methodology to predict authenticity of extra virgin olive oil retail products in the USA. Lipids. 2017:52(5):443-55.

20. Mlcek J, Dvorak L, Sustova K, Szwedziak K. Accuracy of the FT-NIR method in evaluating the fat content of milk using calibration models developed for the reference methods according to Rose-Gottlieb and Gerber. J AOAC Int. 2016;99(5):1305-9.

21. Laurens Lieve ML, Knoshaug EP, Rohrer H, Van Wychen S, Dowe N, Zhang M. Solvent-free spectroscopic method for high-throughput, quantitative screening of fatty acids in yeast biomass. Anal Methods. 2019;11(1):58-69.

22. Ami D, Posteri R, Mereghetti P, Porro D, Doglia SM, Branduardi P. Fourier transform infrared spectroscopy as a method to study lipid accumulation in oleaginous yeasts. Biotechnol Biofuels. 2014;7:12.

23. Kosa G, Kohler A, Tafintseva V, Zimmermann B, Forfang K, Afseth NK, et al. Microtiter plate cultivation of oleaginous fungi and monitoring of lipogenesis by high-throughput FTIR spectroscopy. Microb Cell Fact. 2017;16(1):101

24. Srivastava S, Sadistap S. Data processing approaches and strategies for non-destructive fruits quality inspection and authentication: a review. J Food Meas Characterization. 2018;12(4):2758-94
25. Conzen J-P. Multivariate Calibration — a practical guide for developing methods in the quantitative analytical chemistry. 3rd ed. Ettlingen: Bruker Optik GmbH; 2014. p. 124

26. Eisenstecken D, Stürz B, Robatscher P, Lozano L, Zanella A, Oberhuber M. The potential of near infrared spectroscopy (NIRS) to trace apple origin: study on different cultivars and orchard elevations. Postharvest Biol Technol. 2019;147:123-31.

27. Ghidini S, Varra MO, Dall'Asta C, Badiani A, lanieri A, Zanardi E. Rapid authentication of European sea bass (Dicentrarchus labrax L.) according to production method, farming system, and geographical origin by near infrared spectroscopy coupled with chemometrics. Food Chem. 2019;280:321-7.

28. Sohn M, Himmelsbach DS, Barton FE, Griffey CA, Brooks W, Hicks KB. Nearinfrared analysis of ground barley for use as a feedstock for fuel ethanol production. Appl Spectrosc. 2007;61(11):1178-83.

29. Najbjerg H, Young JF, Bertram HC, Afseth NK, Host V, Kohler A. Highthroughput FTIR spectroscopy of intact HepG2 cells reveals additive and non-additive effects of individual fatty acids when given as mixtures. J Biophotonics. 2013;6(5):446-56.

\section{Publisher's Note}

Springer Nature remains neutral with regard to jurisdictional claims in published maps and institutional affiliations.
Ready to submit your research? Choose BMC and benefit from:

- fast, convenient online submission

- thorough peer review by experienced researchers in your field

- rapid publication on acceptance

- support for research data, including large and complex data types

- gold Open Access which fosters wider collaboration and increased citations

- maximum visibility for your research: over $100 \mathrm{M}$ website views per year

At BMC, research is always in progress.

Learn more biomedcentral.com/submissions 